\title{
Comparison of decentration and tilt between one piece and three piece polymethyl methacrylate intraocular lenses
}

\author{
Ken Hayashi, Hideyuki Hayashi, Fuminori Nakao, Fumihiko Hayashi
}

\begin{abstract}
Background-The extent of the decentration and tilt was prospectively compared between one piece polymethyl methacrylate (PMMA) and three piece PMMA intraocular lenses (IOLs) which were implanted in the capsular bag after performing continuous curvilinear capsulorhexis.

Methods-91 patients underwent a one piece PMMA IOL implantation in one eye as well as the implantation of the three piece PMMA IOL with polyvinylidene fluoride loops in the opposite eye. The length of the lens decentration and the angle of the tilt were quantitated using the anterior eye segment analysis system (EAS-1000) at 1 week as well as 1,3 , and 6 months postoperatively.

Results-The mean length of the decentration in the one piece IOL was smaller than that in the three piece IOL at 1 week $(p=0.0092), 1$ month $(p=0.0044), 3$ months $(p=0.0069)$, and 6 months $(p=0.0010)$ postoperatively. However, no significant difference was found in the degree of the tilt between the two types of IOLs throughout the observation periods.

Conclusion-These results clarified that the one piece PMMA IOL with rigid PMMA haptics implanted in the capsular bag provides a better centration than the three piece PMMA IOL with flexible haptics, whereas the tilt was the same between the two types of IOLs.

(Br f Ophthalmol 1998;82:419-422)
\end{abstract}

When posterior chamber intraocular lenses (PC-IOLs) first began to be used, three piece polymethyl methacrylate (PMMA) lenses were generally favoured. ${ }^{12}$ They were easy to insert since they have flexible haptics made of either polyvinylidene fluoride, polypropylene, or polyimide. However, as the IOL implant techniques into the lens capsule have advanced to now include such techniques as continuous curvilinear capsulorhexis ${ }^{3}$ and endocapsular phacoemusification, ${ }^{4}$ surgeons today tend to prefer one piece PMMA IOL. ${ }^{5}{ }^{6}$ The reason for this is because the one piece PMMA IOL is believed to show a better centration than the three piece PMMA IOL. Experimental studies have also demonstrated the one piece lenses with rigid PMMA haptics to have a better centration $^{7}$ and shape recovery capability after long term compression ${ }^{8}$ than the three piece lenses. Nevertheless, previous clinical studies failed to find any significant difference in the extent of the decentration and tilt between the one piece and three piece IOLs, probably due to either some inaccuracies in the measuring method, a small sample size, or a lack of randomisation. ${ }^{9-11}$

The purpose of this study was thus to examine quantitatively the difference in the extent of the decentration and tilt between the one and three piece PMMA IOLs. In this prospective study, a one piece IOL was implanted in one eye and a three piece IOL was implanted in the opposite eye of the same patient in a randomised manner.

\section{Patients and methods}

One hundred patients who were all scheduled to undergo phacoemulsification and IOL implant surgery for the both eyes were voluntarily enrolled in this randomised prospective study. The preoperative exclusion criteria were (1) cataracts due to aetiologies other than age related changes, (2) a history of previous ocular surgery or inflammation, (3) eyes with any retinal pathologies, (4) eyes with pseudoexfoliation syndrome, (5) eyes from patients with diabetes mellitus, and (6) eyes with a small pupillary diameter after a full dilatation of less than $6.0 \mathrm{~mm}$. Before operation, all enrolled patients were randomly divided into two groups by the sealed envelope method including (A) the group implanting one piece PMMA IOL (MZ60BD; Alcon Surgical Inc, Fort Worth, TX, USA) in the left eye and three piece PMMA IOL (UV22 or UV25T; Menicon Co Ltd, Nagoya, Japan) in the right eye, and (B) the group implanting the one piece IOL in the right eye and the three piece IOL in the left eye. The MZ60BD is a one piece biconvex PMMA lens with $6.0 \mathrm{~mm}$ optic and PMMA haptics. The UV22 and UV25T are three piece planoconvex PMMA lenses with $6.0 \mathrm{~mm}$ optics and polyvinylidene fluoride haptics. The total length of these IOLs are 12.5 $\mathrm{mm}$ in the MZ60BD and UV22, and $13.0 \mathrm{~mm}$ in the UV25T (Fig 1). In the 91 eyes of the three piece IOL group, 55 eyes had the UV22 implanted, while 36 eyes had the UV25T implanted.

The MZ60BD is a biconvex one piece PMMA lens with a $6.0 \mathrm{~mm}$ round optic. The UV22 and UV 25T are planoconvex PMMA lenses with a $6.0 \mathrm{~mm}$ round optic. The total IOL length is $12.5 \mathrm{~mm}$ in the MZ60BD and UV22, and $13.0 \mathrm{~mm}$ in the UV25T.

All surgeries were performed by the same surgeon $(\mathrm{KH})$. The surgical procedures used in this study have all been previously 
Table 1 Patient characteristics

\begin{tabular}{llll}
\hline & One piece IOL & Three piece IOL & p Value \\
\hline Number & 91 & 91 & - \\
Age & $69.3(10.5)$ & $69.3(10.5)$ & - \\
Sex (M/F) & $37 / 54$ & $37 / 54$ & - \\
Left/right & $46 / 45$ & $45 / 46$ & $0.8821^{\star}$ \\
Area of the capsulorhexis & $28.70(4.99)$ & $29.20(4.99)$ & $0.4399^{\star}$ \\
\hline
\end{tabular}

^No statistical significance.

Table 2 Postoperative changes in the length of the decentration ( $\mathrm{mm}$ )

\begin{tabular}{llll}
\hline & One piece IOL & Three piece IOL & p Value \\
\hline 1 week & $0.25(0.19)$ & $0.33(0.22)$ & 0.0092 \\
1 month & $0.24(0.15)$ & $0.31(0.17)$ & 0.0044 \\
3 months & $0.27(0.16)$ & $0.34(0.18)$ & 0.0069 \\
6 months & $0.27(0.16)$ & $0.37(0.25)$ & 0.0010 \\
\hline
\end{tabular}

${ }^{\star}$ Statistically significant.

described. ${ }^{12}$ In brief, arch-shaped (frown) incisions, described by Singer, ${ }^{13}$ with a chord length $6.5 \mathrm{~mm}$ were made for the implantation of the $6.0 \mathrm{~mm}$ PMMA optic lens. The surgeon began to make the frown incision $3.0 \mathrm{~mm}$ posterior to the anterior margin of the limbal vascular arcade, and the scleral tunnel was also dissected up to $1.0 \mathrm{~mm}$ anterior to the margin. The continuous curvilinear capsulorhexis, measuring about $5.5 \mathrm{~mm}$ in diameter, was made using a bent 25 gauge needle. After a thorough hydrodissection, endocapsular phacoemulsification of the nucleus and cortical aspiration were performed using the bimanual nuclear cracking procedures. Next, the lens capsule was inflated with $1 \%$ sodium hyaluronate and then the IOLs were inserted into the capsular bag. Finally, the surgeon carefully confirmed whether the IOLs were properly placed in the capsular bag using a push and pull hook.

The length of the IOL decentration and the degree of the tilt were examined using the anterior eye segment analysis system (EAS1000; Nidek Inc, Gamagori, Japan) at 1 week as well as at 1,3, and 6 months postoperatively. The details of the EAS-1000 system has been described in the previous study. ${ }^{14}$ Briefly, the EAS-1000 system is composed of a Scheimpflug charge coupled device video camera unit and an online image analysis computer. The image analysis computer contained a special

One piece IOL

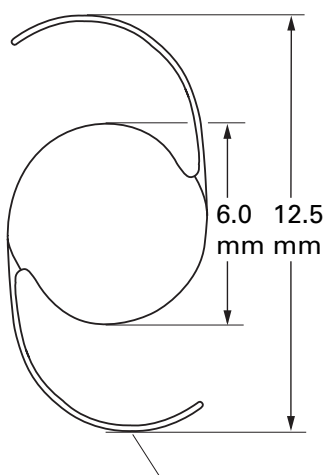

Polymethyl methacrylate (PMMA)

MZ60BD software package to quantitate the length of the decentration (in millimetres) and the degree of the tilt (in degrees) of the implanted IOL. After the full dilatation of the pupil, two Scheimpflug slit images of the IOL were taken at slit angles of $90^{\circ}$ and $180^{\circ}$ using the video camera. After making geometric corrections, these weakly exposed original Scheimpflug slit images were then further enhanced using bicanalisation and curve fitting techniques. Next, by plotting three points on both the anterior corneal surface and the anterior surface of the IOL on these images, the tilt angle of the IOL optic axis relative to the visual axis was calculated by the software package in a three dimensional manner. The length of IOL decentration was also indicated by the distance between the IOL optic vertex from the visual axis. All measurements were carried out by three ophthalmic technicians, who were not informed of the purpose of this study.

Statistical analyses were performed to compare the length of the decentration and degree of the tilt angles between the one piece and the three piece IOL groups. The $\chi^{2}$ test was used for the categoric variables, while the nonparametric Mann-Whitney $U$ test was used for the measurement data. The difference between two values was considered to be statistically significant when the $\mathrm{p}$ value was below 0.05 .

\section{Results}

Of the 100 enrolled patients, four patients in group A and five patients in group B were lost to follow up. As a result, 91 patients remained for analysis. The average patient age was 69.3 (SD 10.5) years, range 38-89 years. The patients consisted of 37 men and 54 women. The patient characteristics are presented in Table 1 . No statistically significant differences were found regarding either the ratio of the left and right eyes or the area of the capsulorhexis. In the first examination after full mydriasis at 1 week after surgery, all IOLs were confirmed to be implanted in the capsular bag.

Table 2 lists the changes in the mean length of the decentration in the one piece and two piece IOL groups. The data are also displayed

Three piece IOL

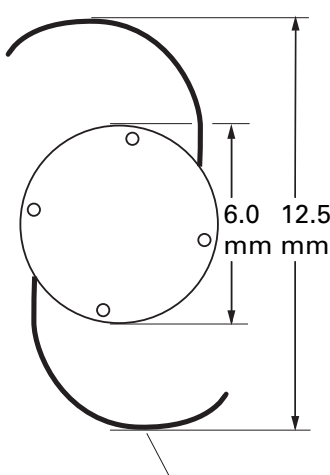

Polyvinylidene fluoride

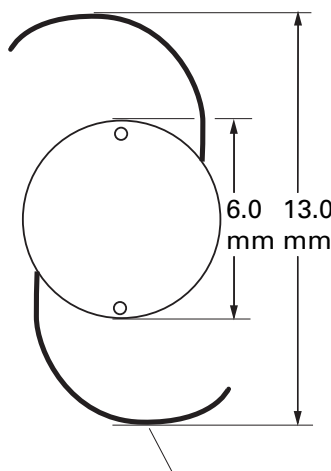

Polyvinylidene fluoride
UV22

Figure 1 Schema of the one piece and three piece intraocular lenses (IOLs) used in this study. 


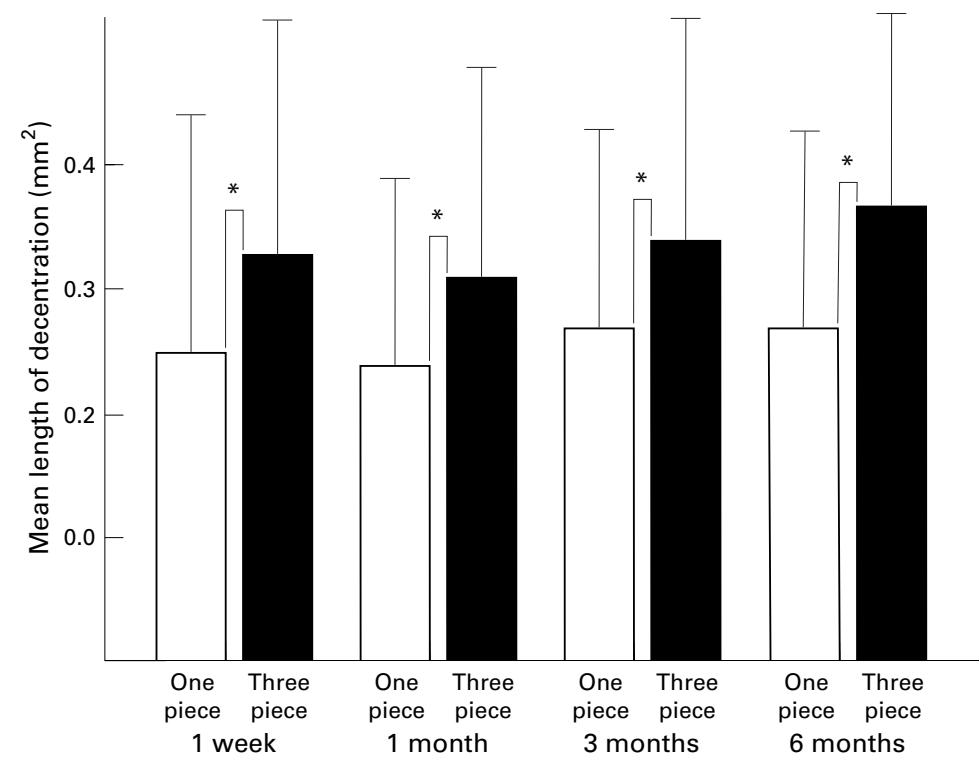

Postoperative period

Figure 2 Comparison of the decentration between the one piece and three piece PMMA IOLs. The mean length of the decentration in the one piece PMMA IOL was significantly smaller than that in the three piece PMMA IOL at 1 week $(p=0.0092), 1$ month $(p=0.0044), 3$ months $(p=0.0069)$, and 6 months $(p=0.0010)$ after surgery. ${ }^{\star}$ Statistically significant.

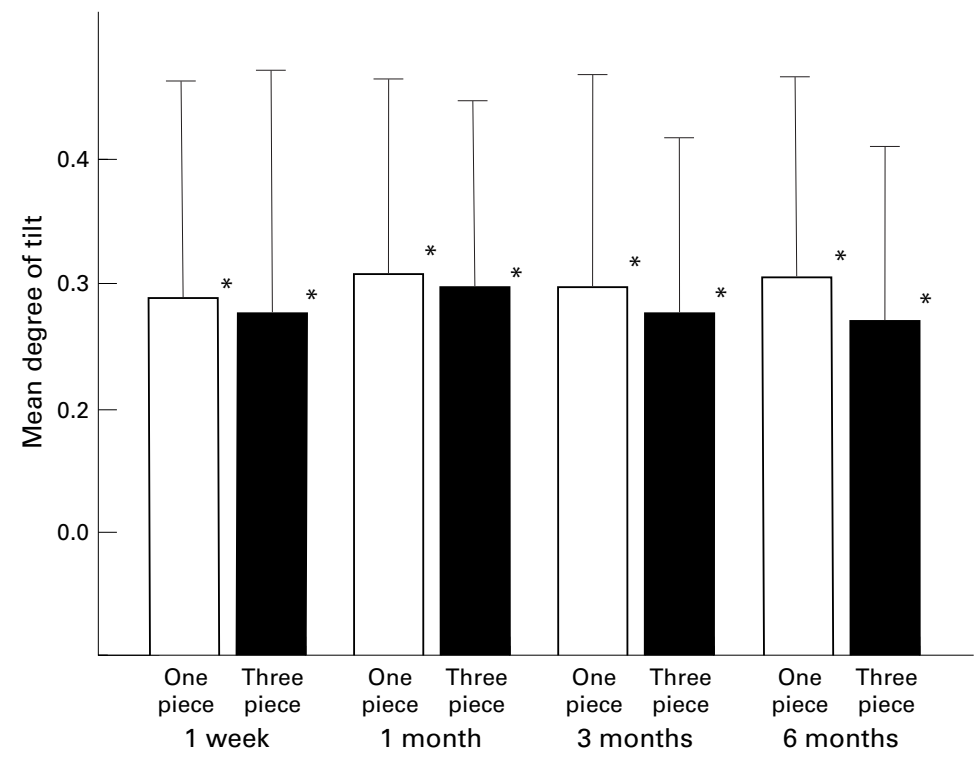

Postoperative period

Figure 3 Comparison of the tilt between the one piece and three piece PMMA IOLs. No statistically significant difference was observed in the tilt angles between the one and three piece IOLs throughout the observation periods. ${ }^{\star}$ No statistical significance.

Table 3 Postoperative changes in the degree of the tilt $\left({ }^{\circ}\right)$

\begin{tabular}{llll}
\hline & One piece IOL & Three piece IOL & p Value \\
\hline 1 week & $2.88(1.74)$ & $2.77(1.98)$ & 0.4791 \\
1 month & $3.10(1.57)$ & $2.98(1.52)$ & 0.6679 \\
3 months & $3.02(1.70)$ & $2.81(1.43)$ & 0.5412 \\
6 months & $3.11(1.62)$ & $2.78(1.41)$ & 0.3129 \\
\hline
\end{tabular}

${ }^{\star}$ No statistical significance.

in Figure 2. Using the Mann-Whitney U test, the mean decentration length in the one piece PMMA IOL group was significantly smaller than that in the three piece PMMA IOL group at 1 week $(p=0.0092), 1$ month $(p=0.0044), 3$ months $(p=0.0069)$, and 6 months $(p=0.0010)$ after surgery.
Table 3 demonstrates the changes in the mean degree of the tilt angles in the two IOL groups. The data are also shown in Figure 3. No significant difference was observed regarding the mean degree of the tilt angles between the two types of IOLs throughout the observation periods. In addition, no significant differences were found in the amounts of the decentration and tilt between the eyes with the UV22 and UV25T lenses.

\section{Discussion}

Experimental studies demonstrated the one piece PMMA IOLs to have better centration ${ }^{7}$ and shape recovery capability against long term compression ${ }^{8}$ than the three piece PMMA IOLs. However, clinical studies did not demonstrate any significant difference regarding lens decentration between the one and three piece PMMA IOLs. ${ }^{9-11}$ The reason for such findings is considered to be due to the fact that these previous studies employed inaccurate measuring methods using two dimensional retroillumination photography to calculate the lens decentration. In contrast, the EAS-1000 system can separately determine the amount of decentration and tilt in a three dimensional manner. The biometrical data obtained by this system have also been confirmed to be both accurate and reproducible. ${ }^{15-17}$

In this study using the EAS- 1000 system, we compared the lens decentration and tilt in the capsular bag after the continuous curvilinear capsulorhexis between the one and three piece PMMA IOLs. These results thus clearly demonstrated the decentration in the one piece IOL to be smaller than that in the three piece IOL throughout the observation periods. On the other hand, no significant difference was found regarding the tilt angle between the two types of IOLs. These results are therefore the first clinical proof that the one piece PMMA lens has a greater tolerance against postoperative shrinkage of the lens capsule, especially in a horizontal direction, than the three piece PMMA lens with flexible haptics.

The IOL centration is considered to be related to many factors in the IOL including lens design, ${ }^{18}$ total lens length, ${ }^{79}$ optic material, ${ }^{14}$ and haptic material. In this study, the one and three piece IOLs have a similar conventional open looped design as well as almost the same total length. In addition, the optics were both $6.0 \mathrm{~mm}$ round-shaped PMMA. The most relevant difference to the decentration between the two types of the IOLs was only the haptic material itself. As a result, our findings clearly confirmed that the rigid PMMA haptic provides better centration compared with the flexible haptic made from other materials. Therefore, the superiority of the rigid PMMA haptics may also be applicable to the foldable lenses with soft material optics.

In this study, both loops of all IOLs were accurately situated in the capsular bag after the continuous curvilinear capsulorhexis without any tears in the anterior capsule. Therefore, strictly speaking, the results obtained in this 
study are considered to hold true only in the case of capsular bag fixation. However, previous studies reported that the IOL decentration was more extensive when the haptics demonstrated either asymmetrical bag/sulcus fixation or out of the bag sulcus/sulcus fixation..$^{20}{ }^{21}$ In addition, other studies have also shown that the IOLs were often decentred in the case of anterior capsule tears. ${ }^{22}$ Based on these findings, we consider that the difference in the decentration and tilt between the one and three piece IOLs will be more prominent in the case of asymmetrical fixation, sulcus fixation, or anterior capsule tears rather than in the case of complete capsular bag fixation. ${ }^{23}$

More importantly, the postoperative capsule shrinkage, which affect IOL decentration and tilt, may be extensive in some pathological eyes. Although the in vivo measurement of the intensity of capsule shrinkage is still impossible to perform, the contraction of the anterior capsule opening is supposed to be closely related to the extent of capsule shrinkage. Many possible risk factors for anterior capsule contraction have been reported. ${ }^{24}$ They included eyes with retinitis pigmentosa, ${ }^{25}$ uveitis, ${ }^{26}$ and pseudoexfoliation syndrome. It is conceivable that the tendency of decentration of three piece lens may thus be enhanced in such pathological eyes. Indeed, we are now investigating the extent of IOL decentration and tilt as well as anterior capsule contraction in these pathological eyes.

In conclusion, the decentration of one piece PMMA IOLs was smaller than that of three piece IOL, whereas the tilt was the same between the two IOLs These findings may imply that the rigid PMMA haptics are better than the flexible haptics made from other materials with regard to the centration of the PMMA optic lenses. Further study is called for to investigate whether the superiority of the rigid PMMA haptics for IOL centration also holds true for foldable IOLs with soft optics.

The authors have no proprietary interest in any of the materials described in this article.

Portions of this article were presented at the 23rd Symposium on Cataract, IOL, and Refractive Surgery, 4-8 April 1997, in Boston, Massachusetts.

1 Apple DJ, Mamalis N, Olson RJ, Kincaid MC. Evolution and classification of intraocular lenses. In: Apple DJ, Mamalis N, Olson RJ, Kincaid MC, eds. Intraocular lenses: evolution, designs, complications, and pathology. Baltimore: Williams \& Wilkins, 1989:11-41.

2 Leaming DV. Practice styles and preferences of ASCRS members-1985 survey. $\mathscr{f}$ Cataract Refract Surg 1986;12: $380-4$
3 Gimbel HV, Neuhann T. Development, advantages and methods of the continuous circular capsulorhexis. If methods of the continuous circula

4 Gimbel HV. Divide and conquer nucleofractis phacoemulsification: development and variations. f Cataract Refract Surg 1991;17:281-91.

5 Leaming DV. Practice styles and preferences of ASCRS members-1994 survey. F Cataract Refract Surg 1995;21: $378-85$.

6 Oshika T, Masuda K, Hayashi F, Majima Y, Leaming DV. Current trends in cataract and refractive surgery in JapanCurrent trends in cataract and refractive surgery

7 Hansen SO, Solomon KD, McKnight GT, et al. Posterior capsular opacification and intraocular lens decentration. Part I Comparison of various posterior chamber lens designs implanted in the rabbit model. $\mathcal{F}$ Cataract Refract Surg 1988;14:605-13.

8 Kimura W, Kimura T, Sawada T, et al. Comparison of shape recovery ratios of single-piece poly(methyl methacrylate) intraocular lens. $\mathcal{F}$ Cataract Refract Surg 1993;19:635-9.

9 Kimura W, Kumura T, Sawada T, Taguchi H, Yoshida T. Postoperative deviation: comparison of 3 IOL haptic materials. Ipn f Ophthalmic Surg 1993;6:449-52.

10 Marumori M, Iwashita S, Kizaki H, et al. A study of factors in intraocular lens dislocation. IOL 1992;6:297-303.

11 Yoshida S, Meya C, Chikuda M, Obara Y. Decentration of intraocular lens for small incision. $f_{p n} \mathcal{f}$ Clin Ophthalmol 1994;48:644-5.

12 Hayashi K, Hayashi H, Nakao F, Hayashi F. The correlationship between incision size and corneal shape changes in sutureless surgery. Ophthalomology 1995;102: $550-6$.

13 Singer JA. Frown incision for minimizing induced astigmatism after small incision cataract surgery with rigid optic intraocular lens. $\mathcal{F}$ Cataract Refract Surg 1991;17:677-88.

14 Hayashi K, Hayashi H, Nakao F, Hayashi F. Decentration and tilt of polymethyl methacrylate, silicone, and acrylic soft intraocular lenses. Ophthalmology 1997;104:793-8.

15 Sakamato Y, Sasaki K. Accuracy of biometrical data obtained from the NIDEK EAS-1000. Ophthalmic Res 1994;26(Suppl 1):26-32.

16 Hirokane K, Kimura W, Kimura T, Sawada T, Ohte A. Reproducibility of measurement of intraocular lens location in situ with an anterior eye segment analysis system. $\mathcal{F}$ tion in situ with an anterior eye segmen Soc 1996;100:240-5.

17 Baez KA, Orengo S, Gandham S, Spaeth GL. Intraobserver and interobserver reproducibility of the Nidek EAS-1000 anterior eye segment analysis system. Ophthalmic Surg 1992;23:426-8

18 Tetz MR, O'Morchoe DJC, Gwin TD, et al. Posterior capsular opacification and intraocular lens decentration. Part II Experimental findings on a prototype circular intraocular lens design. F Cataract Refract Surg 1988;14: 614-23.

19 Ohmi S, Uenoyama K. Experimental evaluation of posterior capsule opacification and intraocular lens decentration: comparison of intraocular lenses of $12.5 \mathrm{~mm}$ and $14.0 \mathrm{~mm}$ diameter. F Cataract Refract Surg 1993;19:348-51.

20 Hansen SO, Tetz MR, Solomon KD, et al. Decentration of flexible loop posterior chamber intraocular lenses in a series of 222 postmotem eyes. Ophthalmology 1988;95:3449.

21 Legler UFC, Assia EI, Castaneda VE, Hoggatt JP, Apple DJ Prospective experimental study of factors related to posterior chamber intraocular lens decentration. 7 Cataract Refract Surg 1992;18:449-55.

22 Davison JA. Analysis of capsular bag defects and intraocular lens positions for consistent centration. F Cataract Refract Surg 1985;12:124-9.

23 Ando M, Fukado Y, Kora Y, et al. Study of IOL decentration. IOL 1992;6:211-5.

24 Namiki M, Yamamoto N, Tagami Y, et al. Risk factors for anterior capsular shrinkage in intraocular lens implantation. fpn f Clin Ophthalmol 1991;45:1828-31.

25 Nishi O, Nishi K. Intraocular lens encapsulation by shrinkage of the capsulorhexis opening. F Cataract Refract Surg 1993;19:544-5.

26 Hansen SO, Crandall AS, Olson RJ. Progressive constriction of the anterior capsular opening following intact capsulorhexis. F Cataract Refract Surg 1993;19:77-82. 